\title{
Discussion on the Cultivation of Composition Writing Habits of Korean Middle School Students with Chinese Language Teaching - A Case of Korean Middle School in Yanbian Autonomous Prefecture
}

\author{
Qingai Jin \\ Department of Discipline Teaching and Research, College of Education of Yanbian University, Yanji, \\ Jilin, China \\ qajin@ybu.edu.cn
}

\begin{abstract}
Professor Ye Shentaothought that composition teaching focused on the cultivation of students' good habit of autonomous writing. Besides, writingdid not express life emotions without observation, accumulation, consideration and diligent correction. Thus, good writing habit is formed with the promotion of Chinese language quality. For the Korean middle school students using Chinese as the second language, it is essential to develop good writing habits. It is the premise and demonstration of Chinese language quality. Therefore, the work analyzed the cultivation of writing habit of Korean middle school students with Chinese language teaching. The mode of "learning-accumulation-experiencing-unification-qualified" was also used.
\end{abstract}

Keywords:Chinese language, Second language, Teaching, Writing habits.

\section{汉语教学模式的视角下，探讨朝鲜族中学生写作习惯的培养}

\author{
延边州朝鲜族中学为例 \\ 金庆爱 \\ 延边大学师范学院学科教育教研部, 延吉, 吉林, 中国 \\ qajin@ybu.edu.cn
}

【摘要】叶圣陶先生认为, 作文教学观的核 心在于培养学生自主写作的良好习惯。只有 养成观察、积累、思考和勤改的习惯才能将 写作作为一种表达生活情感的方式来对待, 才有可能养成良好的作文习惯, 从而提升汉 语素养。对于汉语作为第二语言的朝鲜族中 学生而言, 良好的写作习惯至关重要, 其既 是提高学生汉语素养的前提, 也是学生汉语 素养的一种体现。本文将从汉语教学模式 “学习一积累一体验一整合一达标” 出发,
分析如何培养朝鲜中学生的写作习惯。

【关键词】: 汉语; 第二语言; 教学模式; 写 作习惯

\section{1. 引言}

【数据来源】: 2014 年 9 月-12 月, 延边大 学 2011 级汉语言专业师范生于吉林省 21 所朝鲜族中学实习期间, 共发放调查问卷 1244 份，实际有效份数为 1210 份，回收率 
为 $97.2 \%$ 。

从汉语教学模式出发培养朝鲜族中学生写 作习惯, 从 “学习一积累一体验一整合一达 标” 五个方面, 分层次、分角度抓住重点, 帮助学生养成观察、积累、思考和勤改的作 文习惯, 从而提高学生的作文水平和汉语素 养。

\section{2. 作文学习, 七字五点要抓牢}

\section{1 作文审题要清晰}

作文不跑题是对作文最基本的要求,而 审清题意是避免跑题的关键。审题, 就是审 读作文要求的每一个字, 要求正确理解题意, 不遗漏任何要求, 不随意改变试题要求。

在课内外阅读时, 留心各种文章的题目, 细心领会题目与文章的联系; 在写各种文体 作文时, 注意练习怎样给文章起个恰当而又 醒目的题目。经过不断练习, 就能逐步掌握 一套属于自己的审题方法, 增强审题能力。

\section{2 作文内容要充实}

内容充实, 就是要言之有物, 持之有据。 写人要形象, 写事要具体, 写景要生动, 写 物要准确。作文一定要表达出自己的真情实 感。写记叙文, 要求完整记叙文中的记叙要 素, 人物和事件具体详细, 着重生动形象的 描写。写议论文, 要求明晰议论要素, 论据 典型充足, 重在深刻透彻地说理。

\section{3 作文结构要合理}

作文结构严谨就是要求作文思路展开 有步骤、有次序, 层次分明, 条理清楚, 段 落的划分和安排恰当, 各个部分布局合理, 有头有尾, 有过度有照应。而考场作文, 则 要求学生在构思立意、行文结构、素材运用、 遣词造句等方面有所突破。

\section{4 作文语言求通顺}

语言通顺, 要求作者思路清晰并反复修 改。作文写完了, 一定要看看、读读, 把不 通顺处改正过来。无论哪种体裁的作文, 语 言都要力求简练。哪怕单从语言上删改, 这 样练习几次, 语言的功力也很可能会有明显 的提高。

\section{5 作文书写求工整}

字迹是作文的脸面, 同样不容忽视, 因 此平时一定要挤一些时间练字。考场上至少 需要做到两点: 一是给作文留足够的时间 （一般是考试时间的 (45\%-50\%) ; 二是要 重视字迹, 在阅卷老师面前展示的每一个字 都要写得易认、工整、漂亮。

\section{3. 作文积累, 积累引导运用好}

在问题 “你读作文时是否有摘抄的习 惯？”中学生选 “A.经常摘抄” 的百分比 为 $53 \%$, “B. 偶尔摘抄” 为 $28 \%$ “ C. 在老师 的要求下摘抄” 为 $19 \%$ 。在阅读书籍的过 程中, 有摘抄习惯的学生要比随便翻翻的学 生记住的内容更多, 俗话说 “好记性不如烂 笔头”, 多动笔, 多摘抄, 是进行有效阅读 的绝佳方法。做好摘抄, 对好词好句好段能 反复咀嚼自我消化, 体现到自己的作文中, 达到摘抄的目的。针对学生在作文积累上的 不足, 教师和学生应该注意以下几个方面:

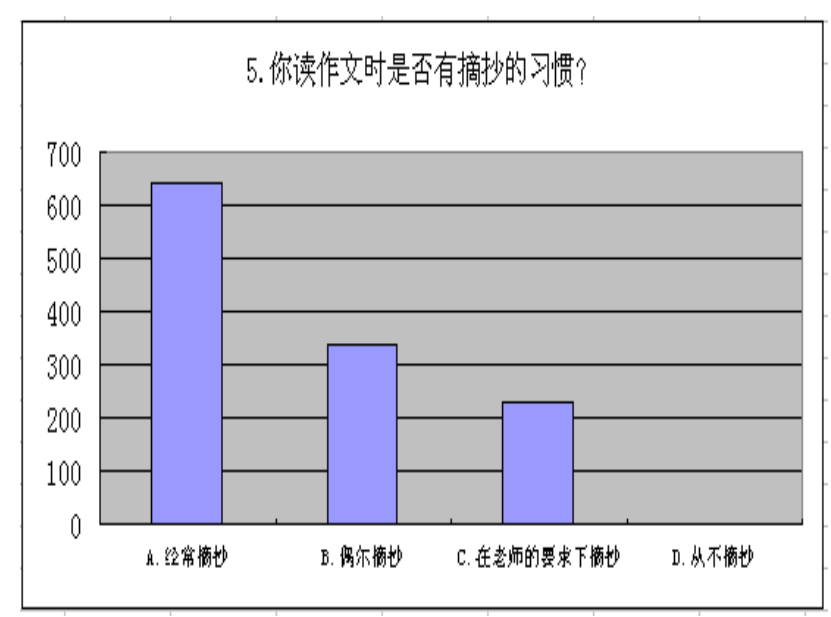

\section{1 积累语言}

学生积累的语言需要进行自我消化, 经 过反复练习, 才能够做到准确的运用。因此, 首先要做到积累与理解并重, 这是学生运用 语言的前提。这一方面, 我们可以通过以下 途径来展开: 一是在阅读教学中进行语言积 累; 二是通过学生模仿运用, 培养遣词造句 的能力; 三是在课堂中举一反三, 让学生逐 步吸收消化。其次要引导学生对积累的语言 进行赏读与评析, 只有学生真正学会运用积 
累的内容, 才算得上是语言积累。在作文时, 我们要在学生中倡导用自己的话去写自己 的真实感受, 在作文中尽情地展示出自己的 特性。

\section{2 积累素材}

引导学生对素材进行积累, 需要利用学 生的情感迁移来积累, 应自觉地培养学生对 作文的兴趣, 根据学生的情感特点, 让学生 在积极愉快的生活中积累素材, 才会在他们 的心中留下深刻的印象。同时要开展丰富多 彩的课外活动来积累学生的素材, 这样做既 开阔视野, 又丰富生活。

\section{3 积累写作方法}

在学生的积累中, 除了语言和素材, 写 作方法的积累也是至关重要的。许多的写作 方法在课堂通过教师教学, 潜移默化的逐渐 积累以后, 我们可以引导学生多读一些课外 美文, 让他们在最直接的感受中体会方法, 并把这些学到的写作方法, 运用到自己的写 作实践中 [1]。

\section{4. 作文体验, 富有个性敢创造}

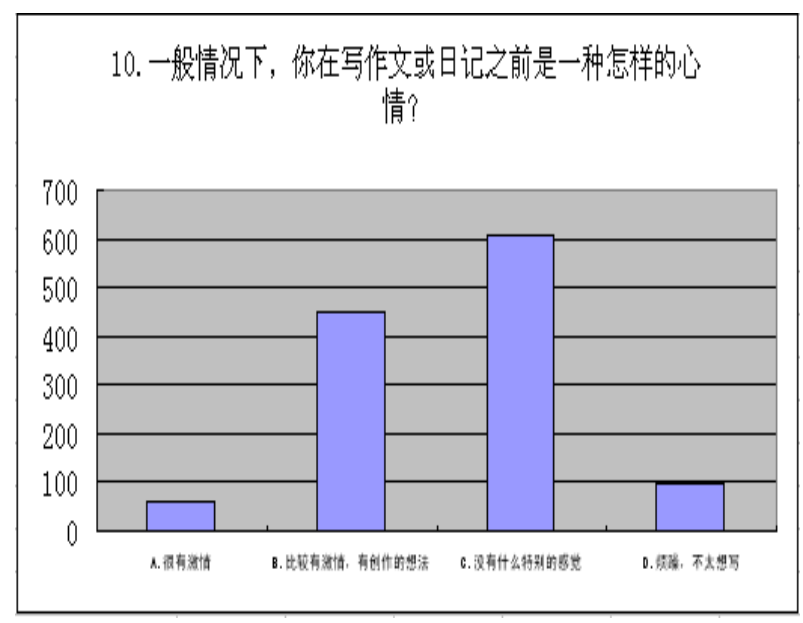

在问题 “10. 一般情况下, 你在写作文 或日记之前是一种怎样的心情? ” 中, 令人 唏嘘的是, 选择选项: “A. 很有激情” 的学 生只有 $5 \%$, 而选择 “ $\mathrm{B}$. 比较有激情, 有创 作的想法” 和 “C. 没有什么特别的感觉” 选 项的各占 $50 \%$ 和 $37 \%$, 而学生对作文的排 斥也表现在了选择 “D. 烦躁, 不太想写” 的 $8 \%$ 上。

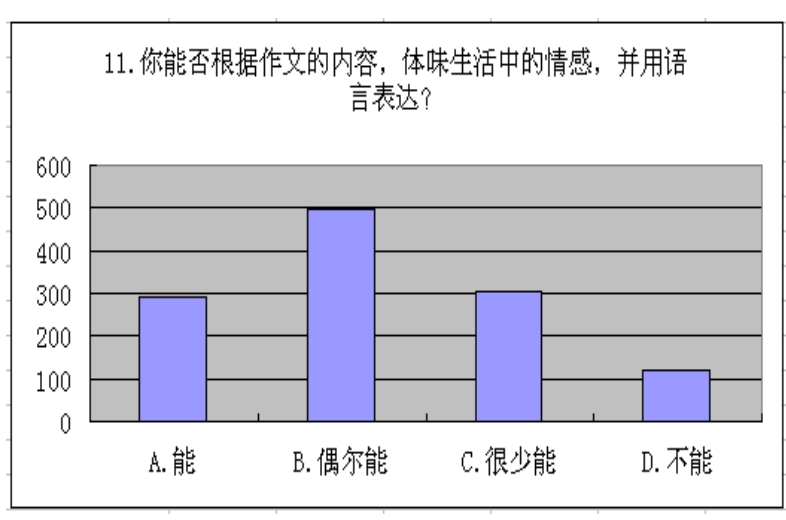

在关于学生对作文的具体操作问题上, 在问题 “ 11 .你能否根据作文的内容，体味 生活中的情感, 并用语言表达” 中, 学生选 择 “A.能” 的占 $24 \%$, 选择 “B.偶尔能” 的是 $41 \%$ ，选择 “C. 很少能” 的为 $25 \%$, 选择 “D.不能” 的有 $10 \%$ 。学生之所以在体 味生活中情感上存在问题, 主要因为中学生 的生活相对枯燥, 学校和家庭两点一线、反 反复复的生活, 让学生没有收集材料、积累 材料的意识和能力。针对学生在作文体验上 的不足, 教师和学生应该注意以下几个方面:

\section{1 体验自己的话语魅力}

良好的语言表达能力是需要不断引导 实践来培养的。首先学生对所学的知识感兴 趣, 才能保持良好的学习状态。同时, 教师 还要善于引导学生在解读中有所感悟, 在理 解文本的基础上, 学生才能说出有条理、有 逻辑的语言, 才能用自己的话语来说事。

\section{2 体验自己的情感观察}

我们在要求学生作文时, 除了选题要贴 近学生外, 更主要的是关注学生的情感体验。 抓住契机, 激活学生的积累内容, 真正达到 “我要写” 的境界。这样的写作练习, 才能 使学生的表达能力得以提高, 学生习作才更 有生命的律动。

\section{3 体验自己的个性创作}

要使学生在作文中展现自己的个性, 对 学生进行创新思维的训练是必不可少的。我 们知道, 创新思维具有三个特性: 就其思维 的广度而言，具有发散性; 就其思维的灵活 性而言, 具有变通性; 就其思维的个性而言, 具有独特性。 


\section{5. 作文整合, 激发情感促习惯}

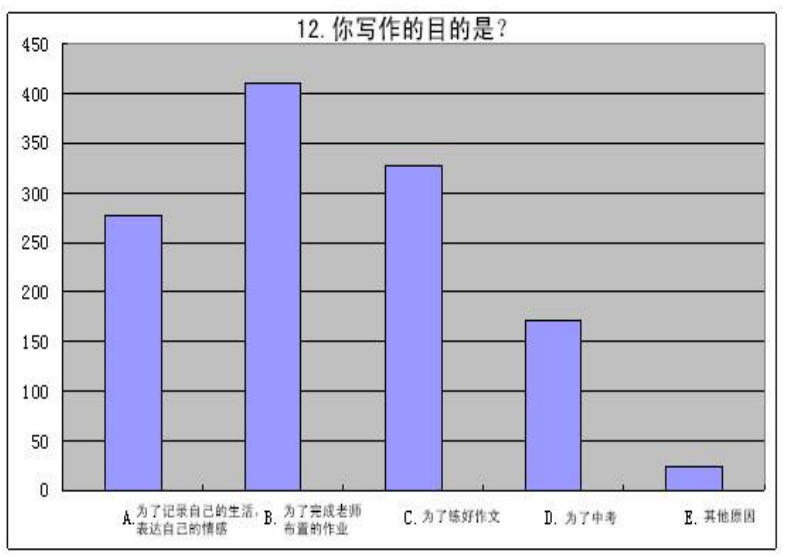

在问题 “12. 你写作的目的是?” 中选 择选项 “A. 为了记录自己的生活, 表达自己 的情感” 的学生比例为 $23 \% ， “ \mathrm{~B}$. 为了完 成老师布置的作业” 为 $34 \%$, “C. 为了练好 作文”为 $27 \%$ ，“D. 为了中考” 为 $14 \%$, 还有 $2 \%$ 的学生选择最后一个“E. 其他原因”。 写作目的的偏差对学生提高作文水平产生 不利影响。针对学生在作文整合上的不足, 教师和学生应该注意以下几个方面:

\section{1 作文整合扶着体验快乐写作}

对于朝鲜族学生而言, 汉语作为第二语 言, 他们既没有大量语言积累, 也不善言辞 表达, 写作的技巧更谈不上高深。动力性障 碍, 是影响初中学生提高写作水平的重要因 素[2]。我们从培养学生的兴趣入手, 通过 以说带写、从仿到写的引导, 循序渐进, 让 学生在体味成功的喜悦中, 逐渐喜欢上写作, 从而逐步提升他们的写作能力。使学生树立 正确的作文价值观, 提高写作理论素养 [3]。

\section{2 作文整合尝试探寻作文奥秘}

为体现学生的主体地位, 在实践中, 我 们初步探索出一种 “从学生中来” 到 “学生 中去” 的 “以学定教” 的习作指导策略, 把 写作前的观察与构思, 拟稿与初步的修改完 全交给学生, 让学生在课外, 甚至在校外, 在放松、自由的环境中完成写作。让学生在 独立自主地进行观察、构思、拟稿、自改、 互改的过程中, 发挥学生的创造性。这样“从 学生中来” 的有针对性的具体形象的习作指 导, 学生很容易接受。当然, 这样指导下的 学生习作, 绝不会千篇一律。

\section{3 作文整合放手感受写作快感}

要积极倡导让作文成为学生生活的需 要, 努力把作文训练与综合实践活动统一起 来。与各科教学紧密联系, 并渗透到学生各 自的校内外生活之中。努力引导学生向自然、 向社会接近, 与社会实践相结合, 把触角延 伸到生活中, 充分发挥作文的工具作用, 让 学生在放手写的过程中, 充分感受到写作的 快意。

\section{6. 作文达标, 内化学生汉语素养}

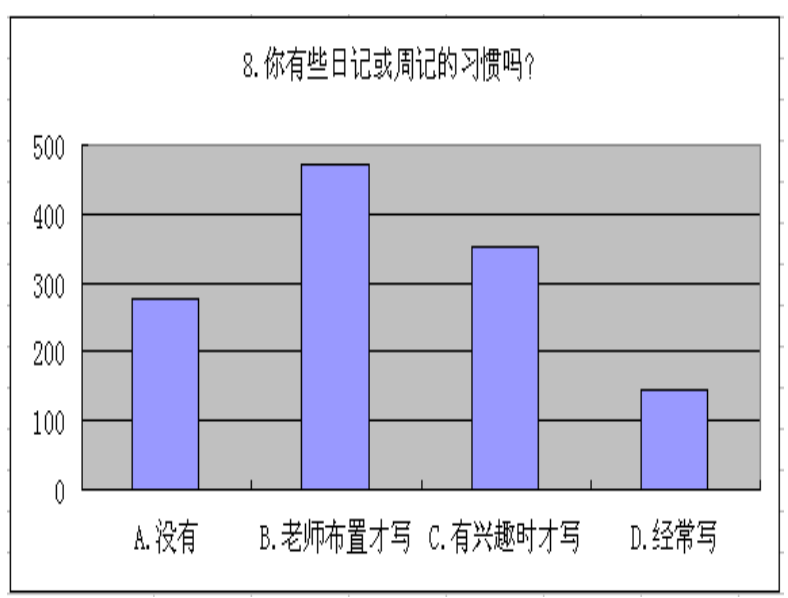

在问题“ 8 . 你有写日记或周记的习惯吗?” 中选择比率最高的是 “老师布置才写”。写 周记是一种非常有益的习惯, 它可以促进师 生情感的交流, 提高学生的写作水平, 还可 以培养学生的思想素质和品格。

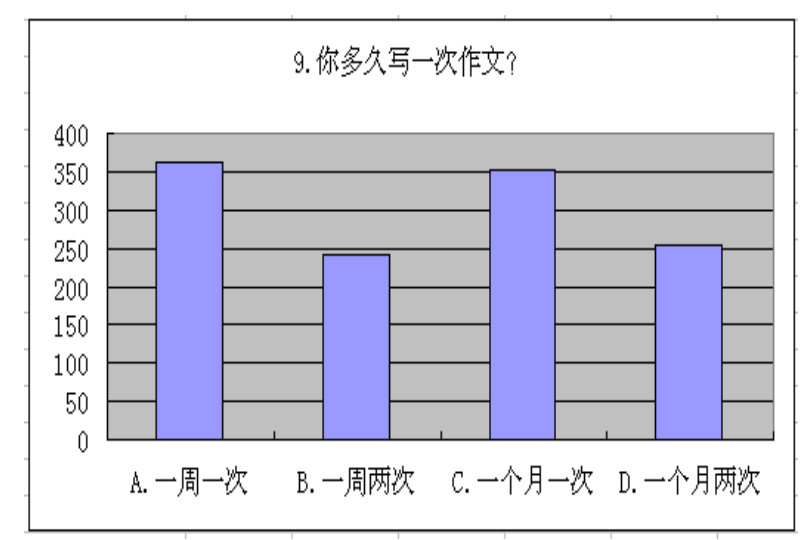

在问题 “9. 你多久写一次作文? ”中 选择比率最高的是 “A.一周一次” , 不难看 出学生学习的被动性, 这一因素阻碍学生作 文水平的提高和汉语基本素养的形成。 


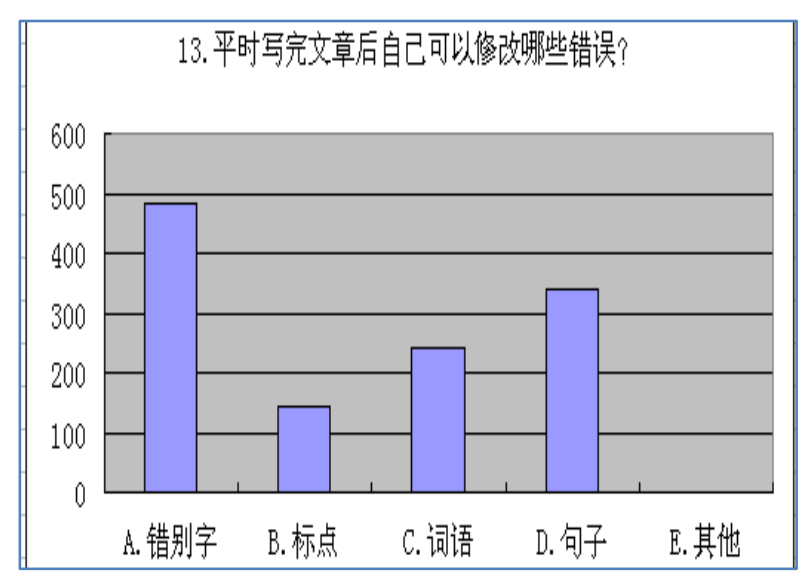

在问题 “13. 平时写完文章后自己可以 修改哪些错误?”中, 选择选项 “A. 错别 字”的学生比例为 $40 \%$, “B. 标点”为 $12 \%$, “C. 词语” 为 $20 \%$ ，“D. 句子”为 $28 \%$, 最后一个 “E. 其它” 选项没有学生选择。 针对学生在作文达标上的不足, 教师和学生 应该注意以下几个方面:

\section{1 培养真实写作的习惯}

真实是写作的生命。缺少真实性的文章 不能表达作者的思想感情[4]。平时在作文 引导时, 要特别注意学生的真实创作, 让学 生养成真实作文的良好习惯。叶圣陶先生认 为在作文教学中, 首先要求学生说老实话, 决不允许口是心非, 可见作文贵在真诚。因 此, 在作文教学中要如实说话, 说真话、露 真情, 才能使读者信服, 才能打动读者的心, 产生情感上的共鸣。

\section{2 培养写作积累的习惯}

一说写作文就有很多学生感到头疼, 感 到下笔困难。究其原因, 无非就是没有什么 东西可以写, 即 “巧妇难为无米之炊”。为 此, 我们必须解决 “无米之炊” 这个困局, 引导学生注意积累, 养成积累的习惯。要强 化积累意识, 只有积累意识增强, 才能自觉 注意积累, 做生活的有心人。

\section{3 培养 “四多” 的写作习惯}

“四多” 分别指多阅读、多练习、多思考和 多修改。多阅读, 多练习, 读书是吸收、积 累的过程, 思想、素材、感受以至语言的表 达方法都在不知不觉中储存起来。“熟读唐 诗三百篇, 不会吟诗也会吟”, “博观而约 取, 厚积而薄发”, 一旦需要, 这些储存就
会蜂拥而至, 跳出来供你应用, 你可以很自 然地随手拈来, 不费思索而顺理成章, 进而 推陈出新, 把自己的情意表达得生动、完美。 多读的确是一条通向学好写作的成功之路。 多思考, 对所见所闻要多思考, 对周围的点 点滴滴要有自己的看法或独到的见解, 写作 时, 养成先构思、列提纲的习惯。这样, 下 笔如有神。多修改, 作文修改的过程是个反 复加工, 反复锤炼的过程, 文章不厌百回改, 越改越精美。从起始年级起, 就应教给学生 自改作文的方法, 调动学生自改作文的兴趣, 多方面培养学生自改的习惯[5]。

\section{7. 结束语}

总之, 汉语教学模式的视角下, 朝鲜族 中学汉语写作习惯有了新思路、新方法, 新 认识。学生作文习惯的好坏, 直接影响到学 生的作文水平。培养良好的作文习惯是写好 作文的前提和基础, 学生有了良好的作文习 惯, 自然能够学得扎扎实实、循序渐进, 写 作能力也自然能够不断提高。进一步发展提 高汉语水平是每位汉语教学工作者的追求, 需要不断完善、提高。

\section{References}

[1] Chen Hanxiang. The cultivation of students'good writing habit with integration of reading and writing in textbooks.Teaching Research of Composition Writing. 2014

[2] Zhang Ying. Teaching research of middle school composition writing. Shenyang Normal University, 2014

[3] Li Meixuan. Research on the effectiveness of teachers' composition writing guidance in middle school. Guangxi Teachers College, 2011

[4] Peng Jinyan; Zhang Shibing. Four writing habits that require cultivation. Literature Education,June, 2008

[5] Huang Yiwen. The Improvement of students' composition writing with good habits . Intelligence, 2014 